\title{
Forecasting Models for Hydropower Unit Stability Using LS-SVM
}

\author{
Liangliang Qiao and Qijuan Chen \\ College of Power and Mechanical Engineering, Wuhan University, Wuhan 430072, China \\ Correspondence should be addressed to Qijuan Chen; qjchen@whu.edu.cn
}

Received 14 January 2015; Revised 30 April 2015; Accepted 7 May 2015

Academic Editor: Michael Small

Copyright ( 2015 L. Qiao and Q. Chen. This is an open access article distributed under the Creative Commons Attribution License, which permits unrestricted use, distribution, and reproduction in any medium, provided the original work is properly cited.

\begin{abstract}
This paper discusses a least square support vector machine (LS-SVM) approach for forecasting stability parameters of Francis turbine unit. To achieve training and testing data for the models, four field tests were presented, especially for the vibration in $Y$-direction of lower generator bearing (LGB) and pressure in draft tube (DT). A heuristic method such as a neural network using Backpropagation (NNBP) is introduced as a comparison model to examine the feasibility of forecasting performance. In the experimental results, LS-SVM showed superior forecasting accuracies and performances to the NNBP, which is of significant importance to better monitor the unit safety and potential faults diagnosis.
\end{abstract}

\section{Introduction}

Hydroelectric power's low cost, near-zero pollution emissions, and ability to quickly respond to peak loads make it a valuable renewable energy source [1]. According to statistics, hydropower provides $22.45 \%$ of the electricity used in China and almost $30 \%$ of the nation's electricity from all renewable sources in 2013 [2]. By the end of 2013, about 273,000 MW of hydropower generation capacity exists in China [3]. More than half of China hydroelectric capacity is in the western provinces of Yunnan, Tibet, and Sichuan, with approximately $57 \%$ of the national total capacity $[4,5]$.

Hydropower generation varies greatly between years with varying inflows, as well as competing water uses, such as flood control, water supply, recreation, and in-stream flow requirements [1]. Given hydropower's economic value and its role in complex water systems, it is reasonable to monitor and protect the hydropower unit from harmful operation modes. A unit is often operated through rough zone which will cause the unit vibration and the stability performance will decline. The accident occurred at 8:13 a.m. on August 17, 2009, at turbine number 2 of the Sayano-Shushenskaya Dam, Russia's largest hydropower plant, which caused heavy casualties and property losses [6]. As [7] states, the main technological causes are that hydraulic unit number 2 often entered the nonrecommended band during startup and shutdown operations and load regulation; what is worse, the unit was under long-term service with inadmissible vibration, particularly during the operation with the temporary turbine wheel, to ensure the stability is ultimately connected with the safety and significant economic efficiency of using hydropower plants as a source of renewable energy.

There are some parameters to describe the unit stability, such as vibration, pressure, and noise. When the parameters exceed a certain value, the unit would run in an instability condition. The serious vibration of rotating parts will cause the shaft misalignment. Excessive vibration of generator rotor will increase the abrasion between slip ring and brush, and the brush would spark. What is worse, the whole plant house and equipment would be damaged when the resonance occurs. The fluctuating pressure in DT will make the flow system oscillate and the pipe wall crack and even the steel plate will be lost. Abnormal noise generated by unit unstable operation will be harmful to the workers' physical and mental health. Existing recommendations in Chinese National Standards regarding stability parameters of hydropower units, GB/T 11348.5-2008 [8] and GB/T 17189-2007 [9], have alarm levels based on statistical data and are often used as an aid to determine and decide if a unit is to be stopped for maintenance. For example, the standards GB/T11348.5-2008 and 
GB/T17189-2007 divide vibration levels into classes with increasing levels from Class A to Class D, where Class A is a good machine that does not need attention while Class $D$ is a machine that should be stopped for immediate corrective action. The permitted levels for each class vary with the unit's rotational speed; a low speed permits higher values of vibration levels in each class, compared to high speed. The standards are not sufficient as vibration monitoring standards since they do not consider the physical properties of bearings and brackets, as well as specific characteristics of a plant [10].

It is an effective way to understand the stability characteristics of a unit by field test under different working conditions. To determine a machine's mechanical condition, Nässelqvist et al. $[10,11]$ used strained gauges installed inside pivot pin to measure the bearing load in a hydropower unit. Talas and Toom [12] studied the accurate measurement and analysis of the dynamic air gap behavior of large hydroelectric generators using a new fibre-optics instrumentation system and the air gap tests were performed on four $184 \mathrm{MV} \cdot \mathrm{A}, 15.6 \mathrm{~m}$ stator bore diameter generators with 16 radial stator support rods. Sun et al. [13] made stability tests for the ALSTOM units on the left bank of the Three Gorge hydropower station under low head and gave suggestions for the operation. Fendin et al. [14] gave a black start test of the Swedish power system, which is focused on voltage control and governor control as well as on the capability of the individual power units. Khodabakhchian et al. [15] performed a more thorough EMTP investigation in which the models and data were adjusted to reproduce recordings from a field test and proposed a test procedure to determine the parameters of a hydraulic turbine model.

For the task of stability parameters identification of a hydropower turbine, it is possible to define a regression vector from a set of inputs and nonlinear mapping in order to finally estimate a model suitable for prediction. There are some typical methods for regression applied in many areas of engineering research [16-18], such as artificial neural network (ANN) and support vector machine (SVM). ANN usually suffers from the existence of many local minima, choosing the number of hidden neurons and determining the structure of the network, the length of the learning cycle, and the type of the learning process [19]. SVM is a relatively novel powerful machine learning method based on statistical learning theory, which was introduced by Shahlaei et al. [20]. The standard SVM is solved by quadratic programming methods which are time consuming and finding the final SVM model can be very difficult because a set of nonlinear equations must be solved [21]. As a simplification, Rubio et al. [22] proposed a modified version of SVM called least square support vector machine (LS-SVM) which resulted in a set of linear equations instead of a quadratic program. LS-SVM has been applied to prediction and classification with promising results, as can be seen in some works [23-26].

In this paper, a method based on LS-SVM model is presented for prediction and regression of hydropower unit stability parameters. The data are obtained from a field test of a $200 \mathrm{MW}$ Francis unit under different working conditions. The results show good performance of the model, which is of great significance to the unit condition monitoring and fault detection.

The rest of the paper is organized as follows: in Section 2 a brief description of LS-SVM is given and in Section 3 how to obtain the data based on a field test is shown in detail and the model for prediction and regression of hydropower unit stability parameters is presented. The results using the proposed LS-SVM model are discussed in Section 4. Finally, some conclusions are drawn in Section 5 followed by Acknowledgment and relevant references.

\section{Methodology}

2.1. Review of LS-SVM. LS-SVM is a modification to SVM regression formulation, proposed by Rubio et al. The main idea is to transform the problem from quadratic programs to solving a set of linear equations. The LS-SVM regression framework can be formulated as follows [23]. Given the data set $\left\{X_{i}, y_{i}\right\}_{i=1}^{l}$, with input vectors $X_{i} \in R^{p}$ and output values $y_{i} \in R$, consider the regression model $y_{i}=f\left(X_{i}\right)+e_{i}$, where $x_{1}, x_{2}, \ldots, x_{l}$ are deterministic points, $f: R_{p} \rightarrow R$ is an unknown real-valued smooth function, and $e_{1}, e_{2}, \ldots, e_{l}$ are uncorrelated random errors with $E\left[e_{i}\right]=0, E\left[e_{i}^{2}\right]=\sigma_{e}^{2}<\infty$. LS-SVMs have been used to estimate the nonlinear $f$ of the form

$$
f\left(X_{i}\right)=W^{T} \varphi\left(X_{i}\right)+b,
$$

where $\varphi\left(X_{i}\right): R^{p} \rightarrow R^{n_{h}}$ denotes the potentially infinite $\left(n_{h}=\infty\right)$ dimensional feature map. The cost function for the data of the LS-SVM model in the primal space is given by

$$
\begin{array}{rl}
\min _{W, b, e_{l}} & P(W, e)=\frac{1}{2} W^{T} W+\gamma \frac{1}{2} \sum_{i=1}^{l} e_{i}^{2} \\
\text { s.t. } & y_{i}=W^{T} \varphi\left(X_{i}\right)+b+e_{i}, \quad i=1,2, \ldots, l .
\end{array}
$$

The formulation includes a bias term, as in most standard SVM formulations, which is usually not the case in the other methods. The relative importance between the smoothness of the solution and data fitting is governed by the scalar, $\gamma$, referred to as the regularization constant. The optimization that is performed is known as a ridge regression. In order to solve the constrained optimization problem, a Lagrangian is constructed:

$$
\begin{aligned}
L\left(W, b, e_{i}, \alpha_{i}\right)= & P\left(W, e_{i}\right) \\
& -\sum_{i=1}^{l} \alpha_{i}\left\{W^{T} \varphi\left(X_{i}\right)+b+e_{i}-y_{i}\right\},
\end{aligned}
$$

where $\alpha_{i}$ is as the Lagrange multipliers. The conditions for optimality are given by

$$
\begin{aligned}
\frac{\partial L}{\partial W} & =0 \longrightarrow W=\sum_{i=1}^{l} \alpha_{i} \varphi\left(X_{i}\right), \\
\frac{\partial L}{\partial b} & =0 \longrightarrow \sum_{i=1}^{l} \alpha_{i}=0,
\end{aligned}
$$




$$
\begin{aligned}
& \frac{\partial L}{\partial e_{i}}=0 \longrightarrow \alpha_{i}=\gamma e_{i}, \\
& \frac{\partial L}{\partial \alpha_{i}}=0 \longrightarrow y_{k}=W^{T} \varphi\left(X_{i}\right)+b+e_{i} .
\end{aligned}
$$

By applying the kernel trick $K\left(X_{i}, X_{j}\right)=\varphi\left(X_{i}\right)^{T} \varphi\left(X_{j}\right)$ with a positive definite kernel, $K$, the dual problem is given by the following set of linear equations:

$$
\begin{aligned}
& \left\{\begin{array}{c}
1_{l}^{T} \alpha=0 \\
\left(\Omega+I_{N} \gamma^{-1}\right) \alpha+b=y
\end{array}\right\} \\
& \Longrightarrow\left[\begin{array}{cc}
0 & 1_{l}^{T} \\
1_{l} & \Omega+\gamma^{-1} I_{l}
\end{array}\right]\left[\begin{array}{l}
b \\
\alpha
\end{array}\right]=\left[\begin{array}{l}
0 \\
y
\end{array}\right],
\end{aligned}
$$

where $y=\left[y_{1}, y_{2}, \ldots, y_{l}\right]^{T}, 1=[1,1, \ldots, 1]^{T}, \alpha=\left[\alpha_{1}, \alpha_{2}, \ldots\right.$, $\left.\alpha_{l}\right]^{T}$, and $\Omega \in R^{n \times n}$ with $\Omega_{i j}=K\left(X_{i}, X_{j}\right)$.

The resulting LS-SVM model can be evaluated at new point $X^{*}$ by

$$
\hat{f}\left(X^{*}\right)=\sum_{i=1}^{l} \alpha_{i} K\left(X_{i}, X^{*}\right)+b .
$$

In (7), $K\left(X_{i}, X_{j}\right)$ is defined as the kernel function. The value of the kernel is equal to the inner product of two vectors, $X_{i}$ and $X_{j}$, in the feature spaces $\varphi\left(X_{i}\right)$ and $\varphi\left(X_{j}\right)$; that is, $K\left(X_{i}, X_{j}\right)=\varphi\left(X_{i}\right)^{T} \varphi\left(X_{j}\right)$. This kernel must be positive definite and must satisfy the Mercer condition.

\subsection{Feedforward Neural Network Using Backpropagation} (NNBP). The feedforward NNBP is a very popular model in neural networks. It does not have feedback connections, but errors are backpropagated during model training. Least mean squared error is used. Many applications can be formulated when using a feedforward NNBP and the methodology is used as the model for most multilayered neural networks. Errors in the output determine measures of hidden layer output errors, which are used as a basis to adjust the connection weights between the pairs of layers. Recalculating the outputs is an iterative process that is carried out until the errors fall below a certain tolerance level. Learning rate parameters scale the adjustments to weights. A momentum parameter can also be used in scaling the adjustments from a previous iteration and adding to the adjustments in the current iteration [23].

2.3. Overfitting in LS-SVM and NNBP. How well the developing models will make predictions for cases that are not in the training set should be put into consideration. LSSVM and NNBP, like other nonlinear parametric models, can suffer from overfitting problem. The models that are too complex may fit the noise, not just the signal, leading to overfitting. Overfitting is dangerous because it can lead to predictions that are far beyond the range of the training data with LS-SVM and NNBP. When the training data include enough information, overfitting can be avoided effectively.
TABLE 1: Specifications.

\begin{tabular}{lll}
\hline Equipment & \multicolumn{1}{c}{ Type } & Parameters \\
\hline & & Rated power: $204.1 \mathrm{MW}$ \\
& Rated head: $107 \mathrm{~m}$ \\
Turbine & HLK333C-LJ485 & Rated speed: $150 \mathrm{rpm}$ \\
& Largest head: $127 \mathrm{~m}$ \\
& Smallest head: $81 \mathrm{~m}$ \\
& Rated discharge: $211.3 \mathrm{~m}^{3} / \mathrm{s}$ \\
\hline & Rated voltage: $13.8 \mathrm{kV}$ \\
& Rated excitation voltage: \\
& $382.48 \mathrm{~V}$ \\
Generator & SF200/40/10800 & Rated excitation current: \\
& & $1301.6 \mathrm{~A}$ \\
& & cos $\Phi: 0.9$ \\
& & Frequency: $50 \mathrm{~Hz}$ \\
\hline
\end{tabular}

In the model applications, the data sets applied in LS-SVM and NNBP models are selected from four field tests, ranging from $0 \mathrm{MW}$ to $200 \mathrm{MW}$ of the whole load. So the training data of the vibration and pressure have covered all the information of the unit, which can deal with overfitting problem of LSSVM and NNBP models.

LS-SVM is based on the structural risk minimization principle, while NNBP is based on the empirical risk minimization principle. LS-SVM includes two structural parts: the error term $\gamma(1 / 2) \sum_{i=1}^{l} e_{i}^{2}$ and the regularization term $(1 / 2) W^{T} W$, seen as (2). This structure can effectively reduce the risk of overfitting. As for NNBP, because the results are based on partially neglecting the regularization term $(1 / 2) W^{T} W$, there is much more danger for overfitting.

In addition, the selection of the kernel function should satisfy the Mercer condition. The radial basis function (RBF) kernel is selected in this paper. LS-SVM with RBF kernel yields a good generalization performance. And using LSSVM with an RBF kernel does not risk too much overfitting, which can be explained by looking to the optimal values of the kernel parameter [27].

\section{Model Applications}

3.1. Data Sets Based on a Field Test. The data sets for the LS-SVM models were selected from field tests of a $200 \mathrm{MW}$ Francis turbine unit in China. The test unit located near the load center of China Eastern Power Grid is mainly used to do the peak and frequency regulation. It was put into power generation on August 16, 2008. Table 1 gives the specifications. The rated power is of $204.1 \mathrm{MW}$ and the rated speed of 150 revolutions per minute (rpm). Its range of working head is between $81 \mathrm{~m}$ and $127 \mathrm{~m}$.

The test will mainly measure the following parameters including frame vibration, guide bearing displacement, and pressure fluctuation in DT. Figure 1 shows the arrangement of measuring points. The capacitance sensor and eddy current sensor were used for the bearing displacement; lowfrequency speed sensor was for the vibration measurement; pressure transmitter was for the pressure fluctuation measurement in DT. Figure 2 shows part sensor installation of 


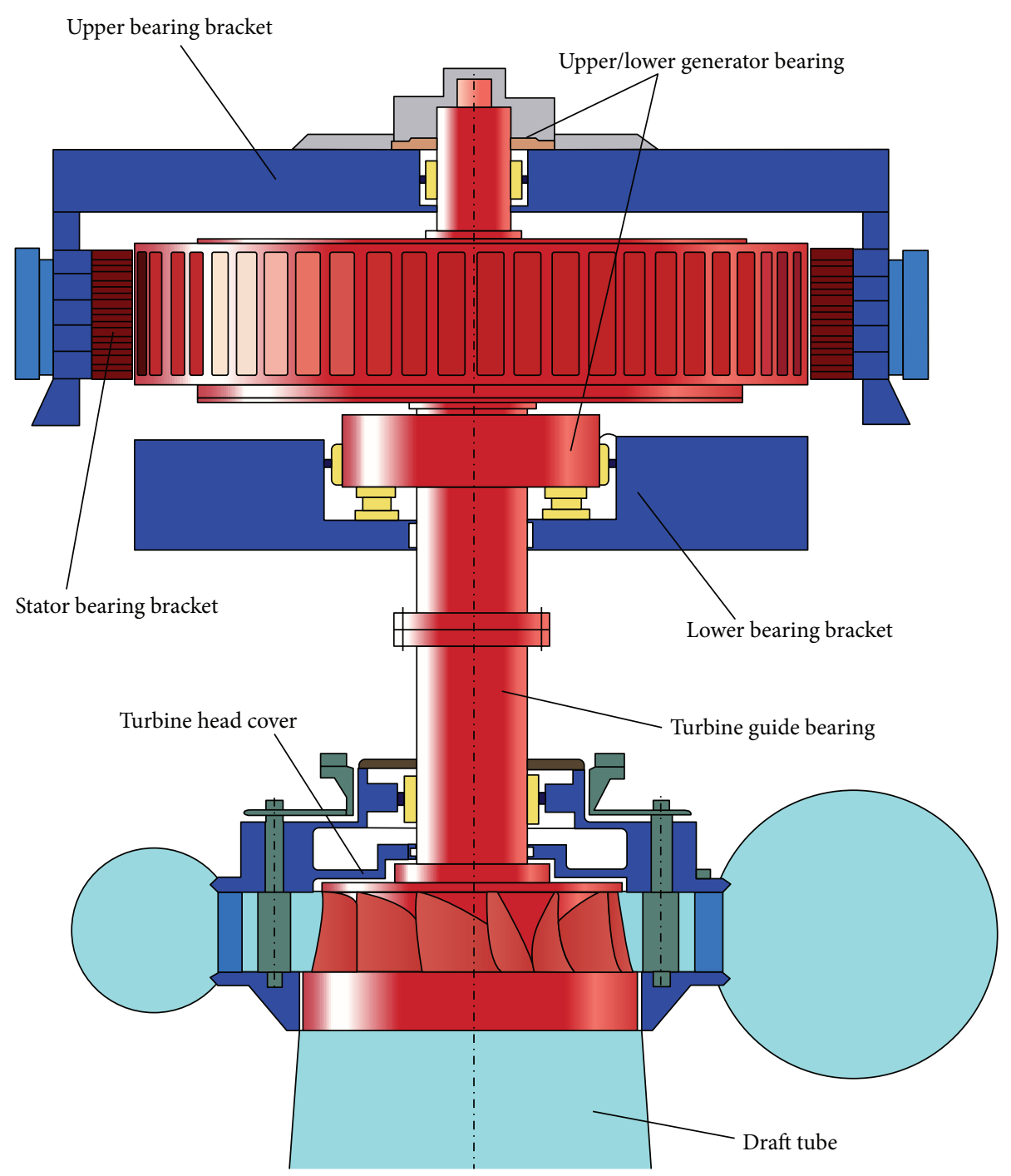

FIGURE 1: Testing components in a hydropower unit.

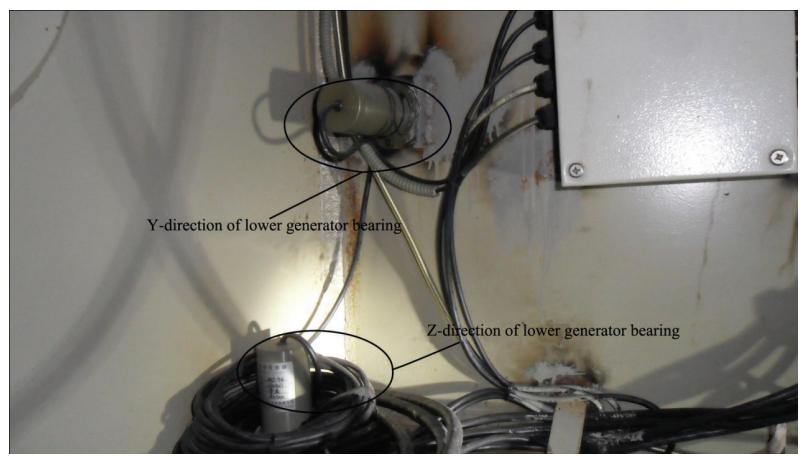

FIGURE 2: Part of the sensor installation of LGB.

LGB. The test working head was $115 \mathrm{~m}, 118 \mathrm{~m}, 120 \mathrm{~m}$, and $122 \mathrm{~m}$. In this paper, we would select the vibration in $Y$ direction of LGB and pressure in DT as the input data of the models.
3.2. Pressure in DT Forecasting. For a Francis turbine, it is significantly meaningful to solve the problem of pressure fluctuation influenced by the low-frequency vortex in DT. Francis turbine works well under the optimal conditions, that is, rated head and wicker gate opening. There is less pressure in DT when the water in runner outlet flows along the axial direction, while, in deviation from the optimal operating conditions, there will be a certain circumferential velocity component for the water flow which will form vortex phenomenon under the action of centrifugal force.

As [28] states, $\Gamma_{2}$ is generally used to describe vortex intensity of the water flow in runner outlet. As $\Gamma_{2}$ is proportional to $V_{u 2}$ ( $V_{u 2}$ is absolute velocity component in the circumferential direction of water flow in runner outlet), it only needs to carry on the research of $V_{u 2}$ which is shown in

$$
V_{u 2}=\frac{\pi r_{a}}{30} \times n-\frac{\cot \beta_{b 2}}{9.8 A_{2}} \times \frac{N}{H \eta},
$$




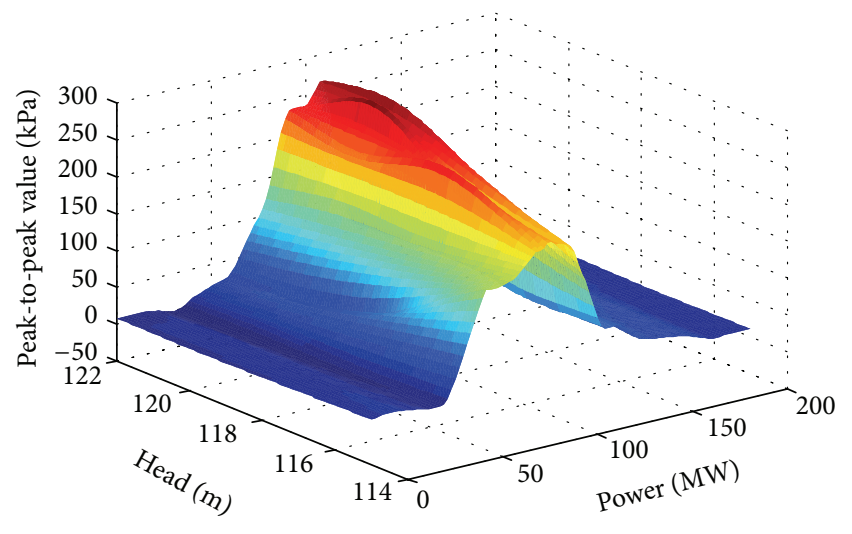

FIGURE 3: Pressure of DT changes with power and head.

where $r_{a}$ is pitch radius of a certain point in runner blade edge; $\beta_{b 2}$ is blade angle; $A_{2}$ is flow section area of runner blade outlet; $n$ is unit rotation speed, rpm; $N$ is the unit output power, $\mathrm{kW} ; H$ is the working head, $m ; \eta$ is unit efficiency.

When $V_{u 2}=0$, turbine works under designed conditions, and water flow in DT enters without crashing; that is, the absolute velocity is perpendicular to tangential velocity. In this case, there is no circular rector in DT and outlet water flow is uniformly distributed. When $V_{u 2}>0$, turbine works under small wicket gate opening. The angle between absolute velocity and tangential velocity is acute and the direction of $V_{u 2}$ is consistent with turbine rotation. $\Gamma_{2}$ is positive. When $V_{u 2}<0$, turbine discharge is bigger than the rated flow and the unit works under big wicket gate opening. The angle between absolute velocity and tangential velocity is obtuse. The direction of $V_{u 2}$ is opposite to turbine rotation. The water flow in DT shows reverse rotation. In a word, when $V_{u 2} \neq 0$, there will be positive or negative circular rector in DT, which is the direct cause of pressure fluctuation.

According to the test results, Figure 3 shows that the average peak-to-peak pressure in access door of DT changes with head and power. In Figure 3, the values range from $10 \mathrm{kPa}$ to $54 \mathrm{kPa}$ in the $0 \mathrm{MW}$ to $100 \mathrm{MW}$ power section; the crest value about $289 \mathrm{kPa}$ appears at $100 \mathrm{MW}$ power in head of $120 \mathrm{~m}$; between $120 \mathrm{MW}$ and $200 \mathrm{MW}$ power section, the values are smaller than $70 \mathrm{kPa}$. A trend can be seen in Figure 3 that the pressure will increase with the head. The value is $148 \mathrm{kPa}$ at head of $115 \mathrm{~m}, 220 \mathrm{kPa}$ at head of $118 \mathrm{~m}$, and $274 \mathrm{kPa}$ at head of $120 \mathrm{~m}$. Through the amplitude-frequency analysis, the dominant frequency is $2.5 \mathrm{~Hz}$ at both lower and higher power section, which is equal to the rotation frequency components. Between $80 \mathrm{MW}$ and $130 \mathrm{MW}$ power region, there is low-frequency vortex signal and the dominant frequency is $0.63 \mathrm{~Hz}$ which is about one-fourth of the rotation frequency components.

Figure 4 gives the time series plot of testing data. Under different working head, the pressure varies with power. As shown in Figure 4, (a) and (b) give the time series of $80 \mathrm{MW}$ and $100 \mathrm{MW}$ power in head of $115 \mathrm{~m}$ and $118 \mathrm{~m}$, respectively. In Figures 4(c) and 4(d), the values are different with $90 \mathrm{MW}$ and $130 \mathrm{MW}$ power in head of $122 \mathrm{~m}$. The values show that nonlinear relationships existed among head, power, and pressure variables.

3.3. Vibration in $Y$-Direction of $L G B$ Forecasting. The vibration data related with power and head were collected on August 16, 2012, September 26, 2012, June 6, 2013, and October 15, 2013, respectively. The LGB is the main loadbearing part of the whole unit. As stated in Chinese National Standards GB/T11348.5-2008 and GB/T17189-2007, there are allowable values for LGB. For example, the radial vibration ( $X$ - and $Y$-direction) is not allowed to be more than $90 \mu \mathrm{m}$ and vertical vibration ( $Z$-direction) no more than $70 \mu \mathrm{m}$. Based on the data analysis of four times field tests, Figure 5 shows that the curve of LGB vibration changes with power and head; Figure 6 displays time series plot of testing data.

Figure 5 shows that the LGB displacement amplitude values change with power and head in $Y$-direction. Displacement amplitude values have no obvious changes with head variation, while the values gradually decrease with the increase of power. In small power region, displacement has its maximum values. When the unit runs in $20 \mathrm{MW}$ and if the head is of $115 \mathrm{~m}$, displacement amplitude value is $46 \mu \mathrm{m}$ in $Y$ direction, and if the head is of $118 \mathrm{~m}$, the value is $45 \mu \mathrm{m}$. Local peak point appears between $90 \mathrm{MW}$ and $140 \mathrm{MW}$. In $120 \mathrm{~m}$ and $122 \mathrm{~m}$ head, the values of local peak point are $40 \mu \mathrm{m}$ and $41 \mu \mathrm{m}$ in power of $130 \mathrm{MW}$ and $110 \mathrm{MW}$, respectively. When the power is close to $200 \mathrm{MW}$, displacement amplitude values are minimal. It is found through spectrum analysis that the dominant frequency of displacement signal is $2.5 \mathrm{~Hz}$ (equal to unit rotation frequency) in small and full power region. And displacement signal appears as $0.63 \mathrm{~Hz}$ of the low-frequency vortex if the power is between $90 \mathrm{MW}$ and $140 \mathrm{MW}$.

The vibration of LGB can be mainly affected by hydraulic, mechanical, and electrical factors. Under different working head, the vibration varies with rotation speed and power. As shown in Figure 6, (a) and (b) give the time series in different rotation speed of $105 \mathrm{rpm}$ and $165 \mathrm{rpm}$. The values vary from $-68 \mu \mathrm{m}$ to $68 \mu \mathrm{m}$ in $105 \mathrm{rpm}$ and $-56 \mu \mathrm{m}$ to $56 \mu \mathrm{m}$ in $165 \mathrm{rpm}$. In Figures 6(c) and 6(d), the vibration values are different with power of $80 \mathrm{MW}$ and $140 \mathrm{MW}$. Also the ranges are shown from $-36 \mu \mathrm{m}$ to $36 \mu \mathrm{m}$ and $-19 \mu \mathrm{m}$ to $19 \mu \mathrm{m}$, respectively. It is difficult to give the precise mathematical model for the relationship between vibration and working conditions.

3.4. Data Set and Software. The data set was divided into two groups: a training set and a testing set. The training and testing sets were applied for the making of the models and to evaluate the predictive authority of the constructed models, respectively. The free LS-SVM toolbox (LS-SVM v-1.8) was applied with MATLAB version R2010a to gather all the LSSVM models.

3.5. Model Performance Evaluation. The statistical means of the mean absolute error (MAE), the root mean square error (RMSE), and the coefficient of determination $\left(R^{2}\right)$ are used for performance measures of the forecasting models in this study. The magnitude of MAE for forecasting a given lead time is a measure of the degree of bias. The RMSE is 


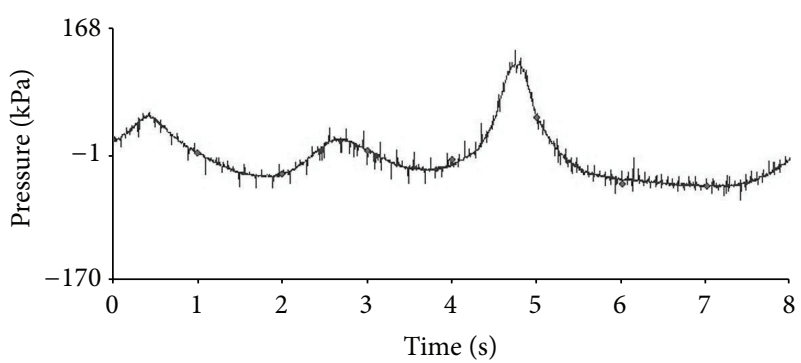

(a) $n=150, H=115$, and $P=80$

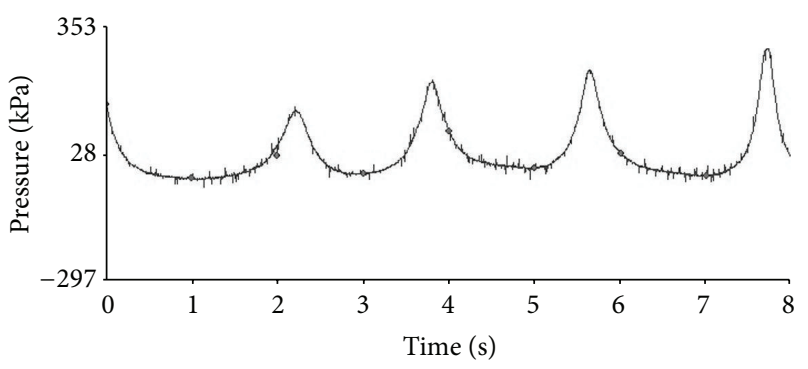

(c) $n=150, H=122$, and $P=90$

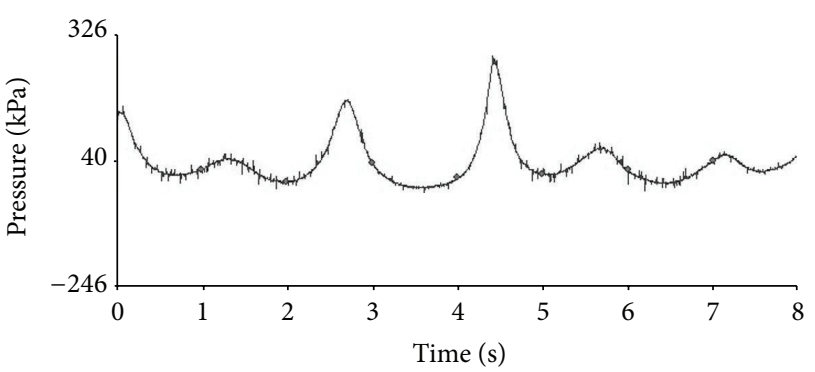

(b) $n=150, H=118$, and $P=100$

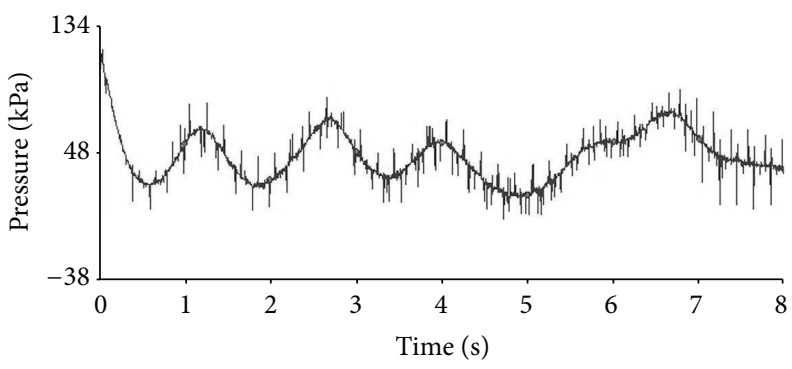

(d) $n=150, H=122$, and $P=130$

Figure 4: Time series plot in access door of DT.

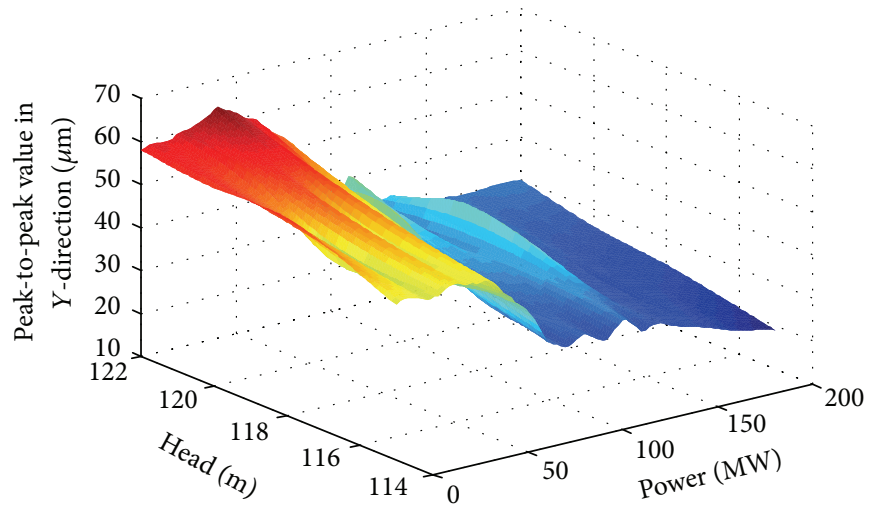

FIgURE 5: Vibration in $Y$-direction of LGB.

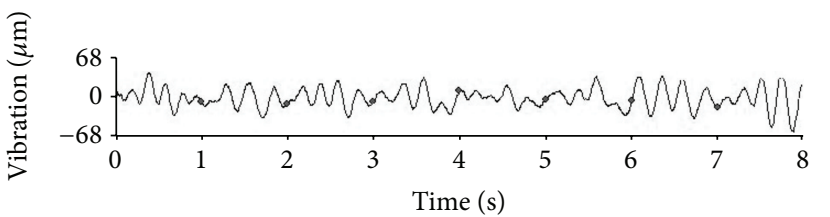

(a) $n=105, H=118$, and $P=0$

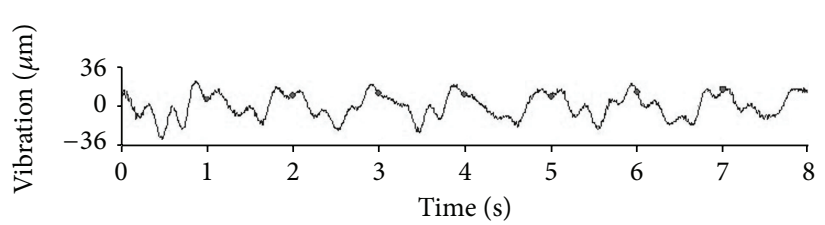

(c) $n=150, H=118$, and $P=80$

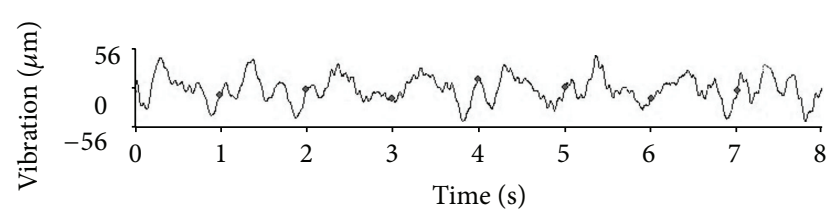

(b) $n=165, H=118$, and $P=0$

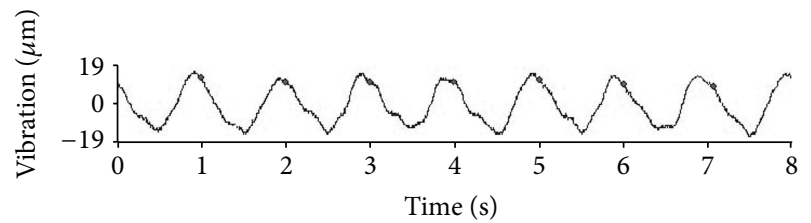

(d) $n=150, H=118$, and $P=140$

FIgURE 6: Time series plot of LGB vibration. 
the average of the forecasting square errors. However, a few large errors can cause a large RMSE value, although most of the forecast error magnitudes are within acceptable limits. Despite this disadvantage, RMSE is useful as an unbiased estimate of the variance of the random component. And a smaller RMSE indicates better forecasting accuracy between two models. These methods can be indicated as follows:

$$
\begin{aligned}
\text { MAE } & =\frac{1}{N} \sum_{i=1}^{N}\left|y_{\text {test }, i}-y_{\text {fore }, i}\right|, \\
\text { RMSE } & =\sqrt{\frac{1}{N} \sum_{i=1}^{N}\left(\left|y_{\text {test }, i}-y_{\text {fore }, i}\right|\right)^{2},} \\
R^{2} & =1-\frac{\sum_{i=1}^{N}\left(y_{\text {test }, i}-y_{\text {fore }, i}\right)}{\sum_{i=1}^{N}\left(y_{\text {fore }, i}-y_{m}\right)},
\end{aligned}
$$

where $y_{\text {test }, i}$ is the predicted value by presented models, $y_{\text {fore }, i}$ is the field test value, $N$ is the amount of input training data, and $y_{m}$ is the average value of the field test data set.

\section{Results and Discussion}

4.1. Vibration Forecasting of $L G B$. Data from the field tests on August 16, 2012, September 26, 2012, and June 6, 2013, under different working conditions were used for training the LS-SVM model. The testing set including 400 pieces of data selected from the test on October 15, 2013, was used to validate the performance of the presented model. In this study, the Gaussian radial basis function was used as the kernel function of LS-SVM. The parameters $\gamma$ and $\sigma^{2}$ are defined as the nonlinear function of the LS-SVM model. $\gamma$ is a regularization constant and $\sigma^{2}$ is the band width of the radial basis function (RBF) kernel. The proper selection of these two parameters is important for the prediction results. Since there are few general guidelines to determine the parameters of LSSVM, this study varied the parameters to select the optimal parameter values for the best forecasting performance. That is, proposed values were chosen over dozens of trial and error experiments. The generalized error was minimum for $\sigma^{2}=0.23$ and $\gamma=10.02$ for LS-SVM. The parameter values presented in this paper may be considered the appropriate level since the sensitivities of SVM parameters relatively are not large, although the appropriate level of parameters may differ according to data. The activation function of the network was a sigmoid function for NNBP.

Figure 7 and Table 2 compare the forecasting performance among the two models with observed and forecasted vibration value in $Y$-direction of LGB. LS-SVM showed excellent performance results for LGB vibration forecasting. The performance of the models was evaluated by the variables which are previously mentioned. The results of the validation test of the forecasting model, as shown in Table 2, clearly showed the greater accuracy of the LS-SVM compared to the NNBP model.

The testing criteria of MAE, RMSE, and $R^{2}$ were calculated in order to measure the forecasting performance. The performance measures of LS-SVM showed lower errors than
TABLE 2: LS-SVM forecasting performance.

\begin{tabular}{lccc}
\hline \multirow{2}{*}{ Models } & \multicolumn{3}{c}{ Performance evaluation } \\
& MAE & RMSE & $R^{2}$ \\
\hline LS-SVM & 2.013 & 2.783 & 0.98 \\
NNBP & 2.154 & 3.012 & 0.93 \\
\hline
\end{tabular}

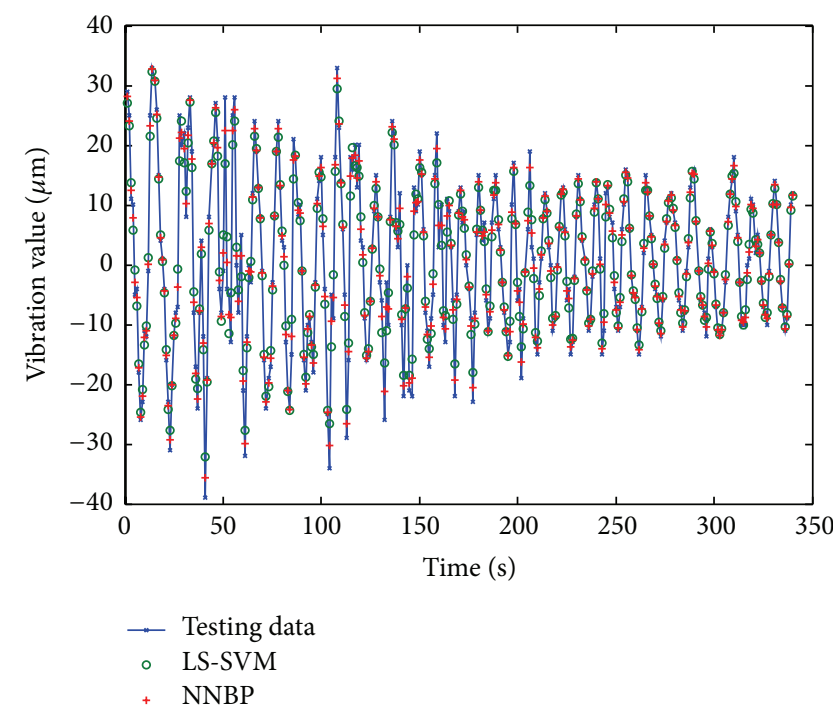

FIGURE 7: Vibration forecasting results in $Y$-direction of LGB.

those of NNBP. The MAE of LS-SVM at 2.013 was lower than the 2.154 of NNBP. The RMSE comparisons showed that the error of NNBP at 3.012 was higher than that of LS-SVM at 2.783. The $R^{2}$ values of the LS-SVM and NNBP were 0.98 and 0.93 which indicated that LS-SVM has higher forecasting ability.

4.2. Pressure Forecasting of DT. Data of pressure in DT from the field tests on August 16, 2012, June 6, 2013, and October 15,2013 , under different working conditions were used for training the LS-SVM model. The testing set including 340 pieces of data selected from the test on September 26, 2012, was used to validate the performance of the presented model. The results of forecasting by LS-SVM were compared with that by NNBP. The optimized obtained values of $\sigma^{2}$ and $\gamma$ were 0.37 and 16.29. The activation function of the network was a sigmoid function for NNBP.

Figure 8 displays a plot of observed versus forecast data to compare the performance between the two models with pressure data of DT. LS-SVM showed excellent performance results for pressure and comparatively good results with respect to peak value matching. The results of the validation test shown in Table 3 clearly indicated that the LS-SVM forecast was more closely aligned to the actual values than the NNBP model, because the forecasting errors in the LS-SVM model were correspondingly smaller than those in the other model.

The test criteria parameters achieved for LS-SVM and NNBP in Table 3 show that the coefficient of determination, 
TABLE 3: LS-SVM forecasting performance.

\begin{tabular}{lccc}
\hline \multirow{2}{*}{ Models } & \multicolumn{3}{c}{ Performance evaluation } \\
& MAE & RMSE & $R^{2}$ \\
\hline LS-SVM & 3.926 & 7.425 & 0.95 \\
NNBP & 4.261 & 7.920 & 0.89 \\
\hline
\end{tabular}

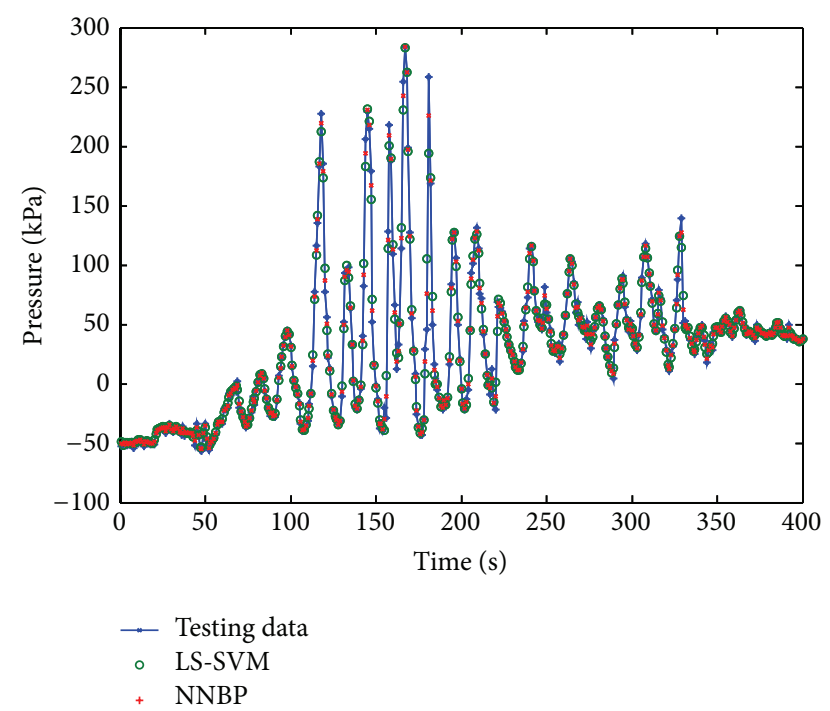

FIGURE 8: Pressure forecasting results of LGB.

MAE, and RMSE values for LS-SVM model are better than NNBP model. The obtained values of $R^{2}$ for LS-SVM and NNBP models were 0.95 and 0.89 , respectively. The MAE of LS-SVM was significantly lower at 3.926 than 4.261 for NNBP, confirming that the variance forecasting error of LS-SVM was smaller than that of NNBP. The RMSE comparison showed that the forecasting error of LS-SVM at 7.425 was lower than that of NNBP at 7.920.

\section{Conclusions}

This paper has presented an LS-SVM approach for forecasting stability parameters of a $200 \mathrm{MW}$ Francis turbine unit. The objective of this paper was to examine the feasibility of using LS-SVM in forecasting the vibration in $Y$-direction of LGB and pressure in DT by comparing it with a heuristic method such as NNBP. And we would clearly verify prediction performance of the models by statistical means of MAE, RMSE, and $R^{2}$. The training and testing data for the models were selected from four field tests, which is an effective way to understand the unit stability characteristics. The field test results indicate that the stability parameters vary with the unit working conditions, such as power, rotation speed, and working head. For better monitoring of the unit safety and potential faults diagnosis, the evaluation of the models had shown that prediction performance of LS-SVM is superior to neural networks using backpropagation in prediction of unit stability parameters data. Future work will aim at extending the methodology developed to deal with more complex unit working condition models and the LS-SVM and NNBP models can be improved tied with optimization algorithm, such as genetic algorithm (GA).

\section{Conflict of Interests}

The authors declare that there is no conflict of interests regarding the publication of this paper.

\section{Authors' Contribution}

Chen Qijuan planned the work and field tests. Qiao Liangliang drafted the main part of the paper and implemented the different forecasting methods, NNBP and LS-SVM. Chen Qijuan contributed to the error analysis.

\section{Acknowledgment}

This research is funded by the National Natural Science Foundation of China (no. 51379160).

\section{References}

[1] K. Madani and J. R. Lund, "Modeling California's high-elevation hydropower systems in energy units," Water Resources Research, vol. 45, no. 9, Article ID W09413, 2009.

[2] China Electric Power Construction Association, 2013 Annual Electric Power Construction Industry, China Electric Power Construction Association, Beijing, China, 2014.

[3] M. H. Wang, Reform and Innovation of Energy, China Energy News, 2014, http://paper.people.com.cn/zgnyb/html/2014-01/ 06/content_1372716.htm.

[4] D. Han, H. W. Fang, B. Z. Yan, and X. Y. Xu, "China's hydropower status in 2013," Journal of Hydroelectric Engineering, vol. 33, pp. 1-5, 2014.

[5] "China commissions 13.9 GW hydropower project," Power, vol. 158, pp. 15-16, 2014.

[6] V. S. Seleznev, A. V. Liseikin, A. A. Bryksin, and P. V. Gromyko, "What caused the accident at the Sayano-Shushenskaya hydroelectric power plant (SSHPP): a seimologist's point of view," Seismological Research Letters, vol. 85, pp. 817-824, 2014.

[7] V. E. Fortov, M. P. Fedorov, and V. V. Elistratov, "Scientific and technological problems of the hydropower industry after the accident at the Sayano-Shushenskaya hydropower plant," Herald of the Russian Academy of Sciences, vol. 81, no. 4, pp. 333340, 2011.

[8] Standardization Administration of the People's Republic of China, Mechanical Vibration-Evaluation of Machine Vibration by Measurements on Rotating Shafts-Part 5: Machine Sets in Hydraulic Power Generating and Pumping Plants, Standardization Administration of the People's Republic of China, Beijing, China, 2008.

[9] Standardization Administration of the People's Republic of China, Code for Field Measurement of Vibrations and Pulsation in Hydraulic Machines (Turbines Storage Pumps and Pump-Turbines), Standardization Administration of the People's Republic of China, Beijing, China, 2007.

[10] M. Nässelqvist, R. Gustavsson, and J.-O. Aidanpää, “A methodology for protective vibration monitoring of hydropower units based on the mechanical properties," Transactions of the ASME, 
Journal of Dynamic Systems, Measurement and Control, vol. 135, no. 4, Article ID 041007, 8 pages, 2013.

[11] M. Nässelqvist, R. Gustavsson, and J.-O. Aidanpää, "Bearing load measurement in a hydropower unit using strain gauges installed inside pivot pin," Experimental Mechanics, vol. 52, no. 4, pp. 361-369, 2012.

[12] P. Talas and P. Toom, "Dynamic measurement and analysis of air gap variations in large hydroelectric generators," IEEE Transactions on Power Apparatus and Systems, vol. 102, no. 9, pp. 3098-3106, 1983.

[13] J. P. Sun, H. Xiong, K. L. Duan, and L. Y. Zheng, "Function analysis of ALSTOM units on the left bank of three Gorge hydropower station operated under low head," Journal of Hydroelectric Engineering, vol. 26, no. 3, pp. 129-133, 2007.

[14] H. Fendin, T. Hansén, M. Hemmingsson, and D. Karlsson, "Black start test of the Swedish power system," in Proceedings of the IEEE PES Trondheim PowerTech: The Power of Technology for a Sustainable Society (POWERTECH '11), Trondheim,Norway, June 2011.

[15] B. Khodabakhchian, G. T. Vuong, and S. Bastien, "On the comparison between a detailed turbine-generator EMTP simulation and corresponding field test results," International Journal of Electrical Power and Energy Systems, vol. 19, no. 4, pp. 263-268, 1997.

[16] Y. S. Diao and H. Ren, "Structural damage early warning based on AR model and factor analysis," Journal of Vibration and Shock, vol. 33, pp. 115-119, 2014.

[17] D.-M. Wang, L. Wang, and G.-M. Zhang, "Short-term wind speed forecast model for wind farms based on genetic BP neural network," Journal of Zhejiang University (Engineering Science), vol. 46, no. 5, pp. 837-841, 2012.

[18] G. D. G. Maria, C. Stefano, F. Antonio, and M. C. Paolo, "Comparison between wind power predction models based on wavelet decomposition with least squares support vector machine (LS-SVM) and artificial neural network," Engergies, vol. 7, pp. 5251-5272, 2014.

[19] V. Dua, "An artificial neural network approximation based decomposition approach for parameter estimation of system of ordinary differential equations," Computers \& Chemical Engineering, vol. 35, no. 3, pp. 545-553, 2011.

[20] M. Shahlaei, A. Fassihi, and L. Saghaie, "Application of PC-ANN and PC-LS-SVM in QSAR of CCR1 antagonist compounds: a comparative study," European Journal of Medicinal Chemistry, vol. 45, no. 4, pp. 1572-1582, 2010.

[21] M. M. Adankon, M. Cheriet, and A. Biem, "Semisupervised learning using Bayesian interpretation: application to LS-SVM," IEEE Transactions on Neural Networks, vol. 22, no. 4, pp. 513524, 2011.

[22] G. Rubio, H. Pomares, I. Rojas, and L. J. Herrera, "A heuristic method for parameter selection in LS-SVM: application to time series prediction," International Journal of Forecasting, vol. 27, no. 3, pp. 725-739, 2011.

[23] S. H. Hwang, D. H. Ham, and J. H. Kim, "Forecasting performance of LS-SVM for nonlinear hydrological time series," KSCE Journal of Civil Engineering, vol. 16, no. 5, pp. 870-882, 2012.

[24] E. Çomak and A. Arslan, "A biomedical decision support system using LS-SVM classifier with an efficient and new parameter regularization procedure for diagnosis of heart valve diseases," Journal of Medical Systems, vol. 36, no. 2, pp. 549-556, 2012.
[25] D. Moreno-Salinas, D. Chaos, J. Manuel de la Cruz, and J. Aranda, "Identification of a surface marine vessel using LSSVM," Journal of Applied Mathematics, vol. 2013, Article ID 803548, 11 pages, 2013.

[26] E. Yilmaz, "An expert system based on fisher score and LSSVM for cardiac arrhythmia diagnosis," Computational and Mathematical Methods in Medicine, vol. 2013, Article ID 849674, 6 pages, 2013.

[27] N. Pochet, F. de Smet, J. A. K. Suykens, and B. L. R. de Moor, "Systematic benchmarking of microarray data classification: assessing the role of non-linearity and dimensionality reduction," Bioinformatics, vol. 20, no. 17, pp. 3185-3195, 2004.

[28] B. Yu and Z.X. Liu, "Research on vibration problem of hydraulic turbines under the high head and low output operation conditions," Journal of Hydroelectric Engineering, vol. 1, pp. 58-65, 2001. 


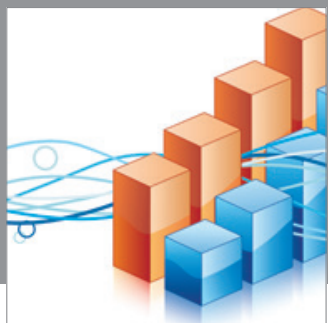

Advances in

Operations Research

mansans

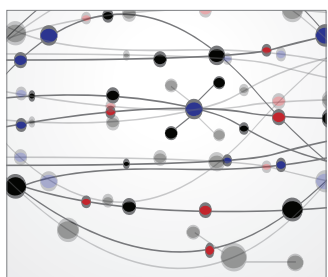

The Scientific World Journal
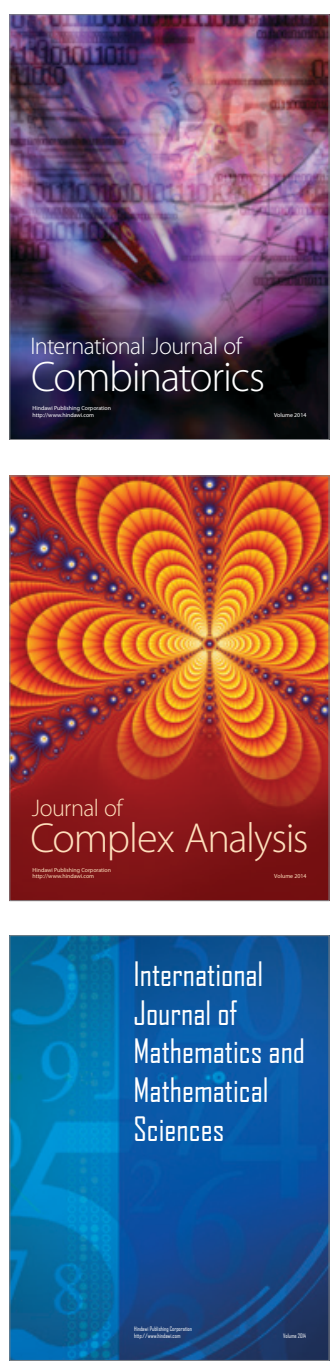
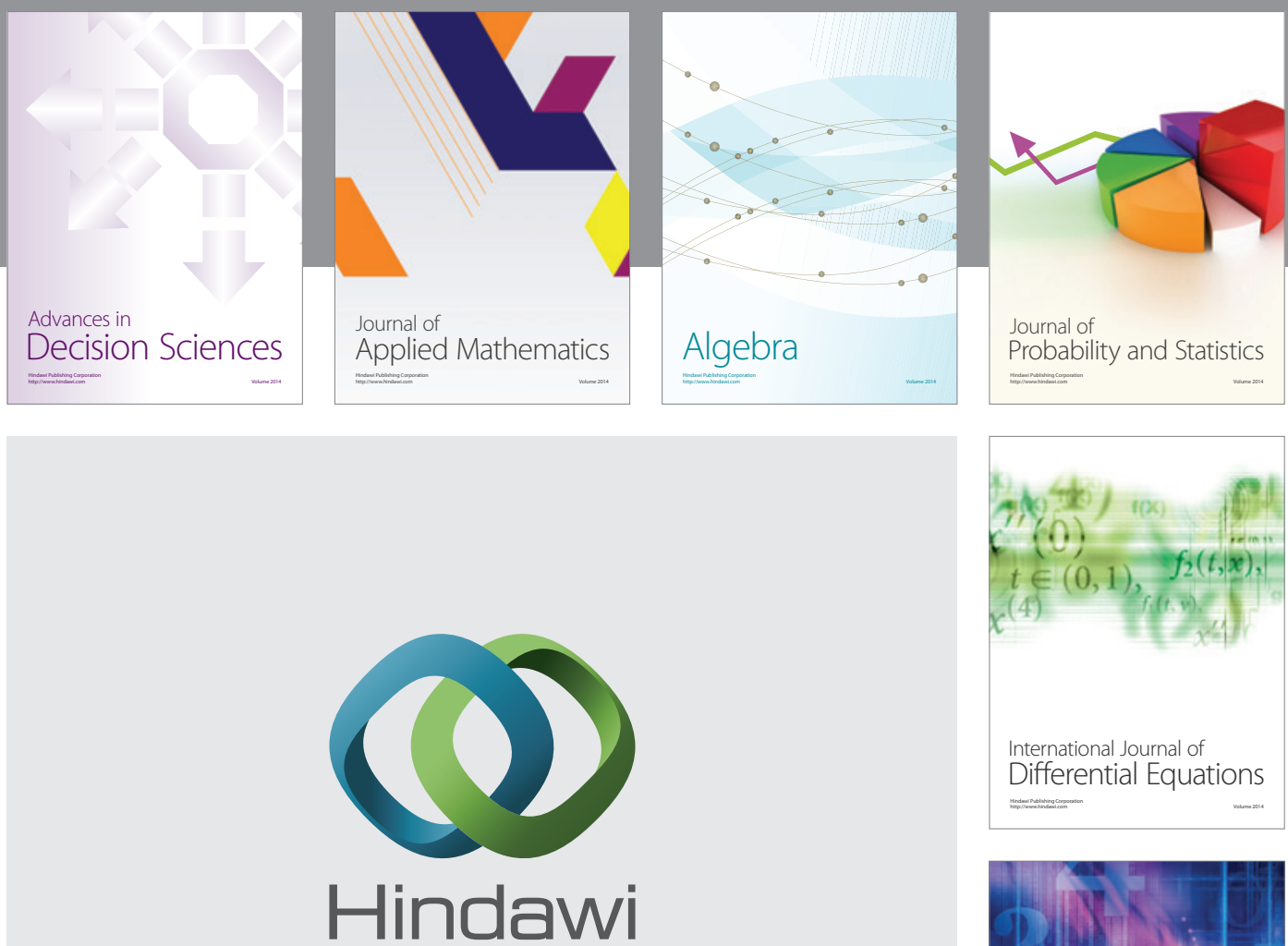

Submit your manuscripts at http://www.hindawi.com
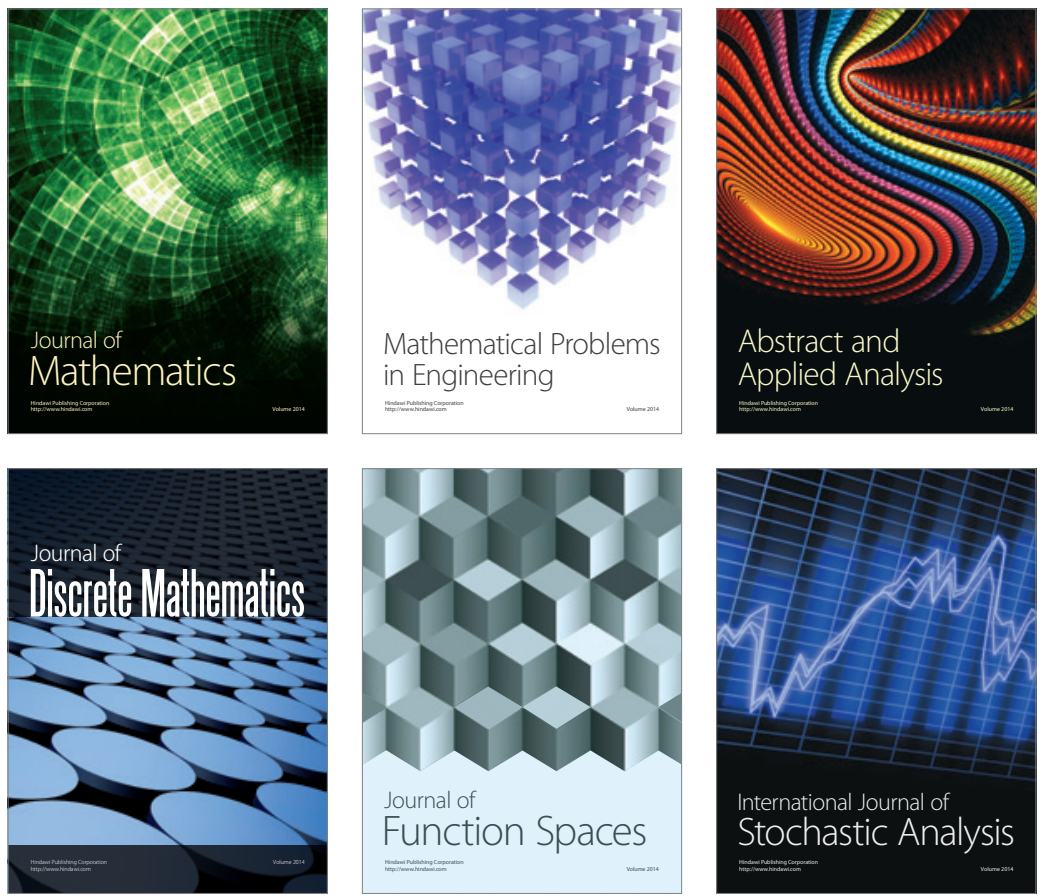

Journal of

Function Spaces

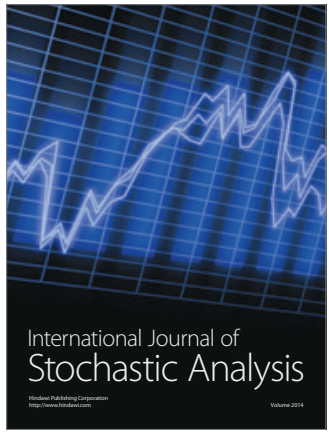

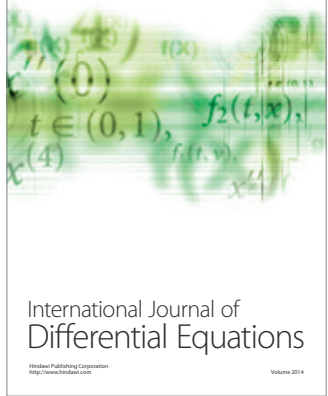
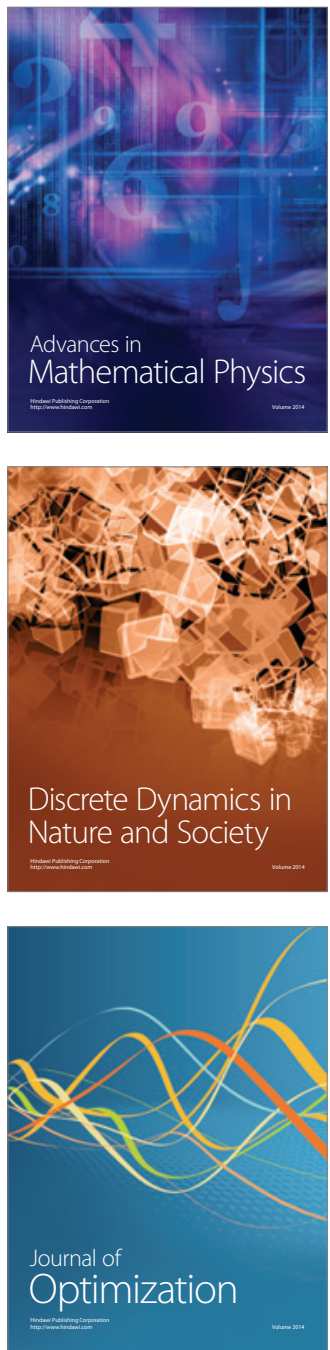